ARTÍCULO DE REFLEXIÓN

\title{
Ejercicios para el empleo de los conectores discursivos en la construcción del texto escrito
}

Exercises for the implementation of discursive connectors

in the construction of written text

\author{
Yarisleidy Castellón Pérez ${ }^{1}$ \\ Israel León Martínez ${ }^{2}$ \\ Kenia González González ${ }^{3}$ \\ Universidad de Ciego de Ávila
}

Recibido: 11.12 .2020

Aceptado: 26.12.2020

\section{Resumen}

El uso adecuado de la lengua materna de forma oral o escrita es de vital importancia en todos los niveles educativos, una parte muy importante para su logro está dado en el empleo de los conectores discursivos en la construcción del texto escrito, en la Educación Preuniversitaria se le brinda un mayor tratamiento a este contenido es por ello que, durante estos meses de aislamiento debido a la pandemia hemos creado e implementado a través de las tecnologías un sistema de ejercicios en función de la aprehensión de los conectores del discurso mediante la construcción textual, el cual demostró ser muy efectivo, no solo perfeccionó la habilidad de construir sino que también se elevó el acervo cultural de las nuevas generaciones.

\footnotetext{
${ }^{1}$ yarilester2018@gmail.com

http://orcid.org/0000-0001-6229-2908

2 israelleonmartinez@gmail.com

http://orcid.org/0000-0002-0184-9183

${ }^{3}$ keniagg@ sma.unica.cu

http://orcid.org/0000-0002-0727-4384
} 
Palabras clave: Sistema de ejercicios, Conectores discursivos, Construcción textual.

\begin{abstract}
The use of the mother tongue orally or the writing is of vital importance in all educational levels, a very important part of its achievement is given in the example of the discourse connectors in the construction of the written text, Preuniversity Education offers a greater treatment to this content and for that reason, during these months of isolation due to the pandemic, we have created and implemented through technology the training system for the understanding of the discourse connectors by means of textual construction, which proved to be very effective, on the ground perfected the ability to build a bell that also raised the cultural collection of the new generations.
\end{abstract}

Keywords: system of exercises, discursive connectors, textual construction.

\title{
1. Consideraciones didácticas sobre el empleo de los conectores discursivos en la construcción del texto escrito en la asignatura español-Literatura en el Preuniversitario
}

La asignatura español-Literatura a través del Programa Director de la Lengua Materna y las Orientaciones Metodológicas, así como el Programa de Humanidades para la Educación Preuniversitaria y demás documentos normativos pone en práctica el proceso de enseñanzaaprendizaje de los conectores y marcadores discursivos en la construcción del texto escrito haciendo uso de los componentes del mismo: objetivos, contenidos, métodos, formas, medios y evaluación del aprendizaje. Debemos tener presente ante todo que la Didáctica tiene como objeto definido el proceso de enseñanza-aprendizaje, por eso es necesario analizar cómo se manifiesta en el uso correcto de los marcadores del discurso porque, ¿quiénes son los que intervienen en la construcción del texto escrito permitiendo el logro de una adecuada coherencia y cohesión?; por su puesto que los elementos coordinantes, relacionantes, subordinantes, los llamados nexos gramaticales, los enlaces extraoracionales, los conectores argumentativos, los procesadores textuales, las partículas, todos estos nombres se refieren a 
un mismo elemento que logra la coherencia y la cohesión en todo texto, "los marcadores discursivos o conectores del discurso".

Los analistas de la lingüística discursiva funcional analizan cómo la elección de determinadas estructuras gramaticales puede estar relacionada con la tipología discursiva, lo anterior quiere decir que, el tipo de contexto condiciona el tipo de texto y las estructuras que se emplean.

La lingüística del texto aborda su objeto de estudio desde tres dimensiones: la semántica, la sintáctica y la pragmática, lo que equivale a la relación significado-forma-contexto, así son asumidas las concepciones de la linguiística discursiva-funcional o lingüística del texto en relación con el estudio de las diferentes estructuras gramaticales.

Una gramática del texto o discurso rebasa los límites de la oración y estudia las relaciones entre las oraciones. La comunicación escrita resulta más compleja para el estudiante que la expresión verbal, ya que; exige el conocimiento de los signos escritos, de las normas para la ortografía, la gramática y la estructuración de las oraciones, por tanto, la construcción del texto escrito constituye un quehacer social y académico y es la macrohabilidad en la que menos competentes se muestran nuestros estudiantes.

En la medida en que todos los niveles se interrelacionan de manera lógica y comprensible, decimos que el texto tiene coherencia; pues constituye una categoría semántica que se refiere a la organización del significado del texto, el cual es desconocido si no se exterioriza, el valor que poseen los signos lingüísticos. El plano expresivo y el contenido, se organizan en niveles jerárquicos que se correlacionan con los correspondientes del contenido: el tema se expresa en el discurso, los subtemas, en párrafos o segmentos, las proposiciones temáticas, en oraciones y los conceptos, en sintagmas.

Determinados autores aluden a que el texto se caracteriza por su cierre semántico y comunicativo, así como por su coherencia profunda y superficial, debida a la intención comunicativa del hablante de crear un texto íntegro, y a su estructuración mediante dos 
conjuntos de reglas: las propias del nivel textual y las del sistema de la lengua. En este sentido, comienzan a configurarse esas características propias del texto que lo definen y permiten su descripción: unidad de sentido (cierre semántico), coherencia, intención del productor textual y estructura.

La expresión linguística designa la propiedad que tienen algunos elementos de la lengua cuando se refieren al espacio, al tiempo y a las personas que concurren en un acto concreto de comunicación, aquí juegan un papel esencial los adverbios. Estos tienen un significado ocasional: solo significan plenamente cuando están actualizados en una situación específica. Halliday, Baena, Parra y otros autores han definido el texto como unidad semántica y coinciden en considerar como texto todo lo que una persona quiere expresar por escrito, que posea una intención y una finalidad en cualquier situación comunicativa escrita, pues durante el ejercicio de construcción del texto escrito se entretejen todos los niveles: el nivel semántico o de significación, el de expresión o formalización lingüística del significado y el fonológico. Por lo tanto, el significado textual se forma lingüísticamente y se exterioriza de forma material como expresión oral o escrita.

En este gran proceso de la construcción textual se pone de manifiesto la complejidad de la actividad constructiva, al respecto Vigotsky planteó que: “...la psicología ha considerado la escritura como una complicada habilidad motora, (...) cuyo dominio representa un punto crítico decisivo en el desarrollo cultural del niño".

Todo texto construido, según apuntan diferentes autores como William Gray, García Arzola entre otros; es portador al menos de tres significados y cito lo expuesto por Rosario Mañalich en el libro "Taller de la Palabra" (1999, p.17):

1-Literal o explícito: Es lo que se expresa de manera directa en el texto.

2-Intencional o implícito: No se dice literalmente, pero se descubre entre líneas, subyace en el texto. Una vez descubierto, se hace también explícito. 
3-Complementario o cultural: Se expresa en la riqueza lírica, profundidad del contenido, cultura general y experiencia del autor reflejada en lo que el texto significa (tratamiento del tema, vocabulario, etc.). Al escuchar a una persona o al leer un escrito, si poseemos un buen nivel de comprensión, estaremos en condiciones de captar tanto lo que el autor dice de manera explícita o de manera implícita, de acuerdo con nuestro universo del saber.

No podemos disociarnos en cuanto a la naturaleza o el carácter del texto escrito y la situación en la que este se construye, todos los elementos que nos rodean se concretan mientras orientamos al estudiante hacia la construcción y determinan considerablemente su importancia, pues en el caso de un texto científico predomina la función representativa o referencial, puesto que el significado se da explícitamente, claro y preciso; lo intencional no se oculta y lo complementario es indispensable conocerlo teniendo en cuenta los términos que se emplean con carácter científico, las fórmulas, las leyes y los principios.

Sin embargo, en el texto literario, es todo lo contrario, lo literal o explícito se descubre de forma intencionada o implícita, es mucho más de lo que la palabra denota, nos interesa lo que connota teniendo en cuenta la función predominantemente artística o poética.

Los marcadores tienen gran importancia en el avance del texto y están en relación con distintas intenciones comunicativas de los hablantes, de igual forma La gramática del discurso como ciencia particular, surge del estructuralismo, pues sus principales representantes, una vez agotadas las posibilidades del análisis de oraciones.

La gramática no puede estudiarse desvinculada de las características de la textualidad, pues nos comunicamos mediante textos o discursos diferentes y ellos se construyen con estructuras gramaticales prototípicas de cada contexto. La gramática del discurso tiene como objetivo el estudio de las estructuras gramaticales en textos o discursos, puesto que nos comunicamos no mediante oraciones aisladas, sino mediante textos o discursos, que tienen en cuenta el contenido que se comunica, la forma con que se hace y el contexto o situación comunicativa en que se emiten, por tanto, debe abordarse cada estructura gramatical, teniendo en cuenta los 
diferentes contextos de comunicación, o sea, los distintos tipos de discursos que se corresponden con las distintas intenciones y finalidades comunicativas de los hablantes.

Con respecto a este tema se hizo una revisión a varias tesis de licenciatura, maestrías y doctorados como por ejemplo la de Adia Gell Labañino (2003) investigó acerca de la construcción de textos escritos en tercer y cuarto grados de la escuela primaria rural, tenemos también a Virgen Onelvis Castellano Borlot (2010), quien se adentró en el mundo de la motivación para aprender a construir el texto escrito argumentativo en los escolares del segundo momento del desarrollo de la escuela primaria. Dentro de las operaciones constitutivas de la escritura que realizan los alumnos se tienen (Domínguez. G. I, 2010):

La planeación, la teatralización o redacción propiamente dicha. La revisión de lo escrito. Como etapas de la actividad de escritura corresponden a la actividad del profesor (sea clase completa o parte de ella:

-Orientación: El maestro motiva, propone y controla.

-Ejecución: El maestro ayuda, propone, precisa, dialoga, escucha, controla.

-Control: El maestro finaliza la actividad, escucha, propone, controla.

Así pues, antes de empezar a escribir, se requiere dedicar una buena parte del tiempo a pensar cómo debería ser el texto que se va a escribir, generando una dialéctica entre lo que sabe del tema, los objetivos que se persiguen y las características de la situación comunicativa, constituyendo esta uno de los subprocesos dentro de la actividad del alumno: la planeación. La didáctica más tradicional ha acuñado los términos de orientación, ejecución y control, para las distintas etapas que integran el proceso de enseñanza - aprendizaje de la construcción del texto escrito. Los doctores Abello Cruz y Montaño Calcines (2012) abordan en el libro “Español-Literatura para escuelas pedagógicas, tomo II”, las cualidades del párrafo y explican a través de un cuadro sinóptico que el texto requiere de una organización mental dada por la coherencia y que puede ser global o local, también requiere de una organización lingüística 
donde se logre la cohesión textual y esta a su vez depende de los conectores, los correferentes y la pronominalización (Abello Cruz y Montaño Calcines, 2012, p. 206).

Para una adecuada construcción del texto escrito es importante tener presentes tres aspectos imprescindibles en el momento en el que se le orienta al estudiante el desarrollo de una construcción textual, ya que, primeramente, debemos motivar, estimular y despertar el interés de los alumnos, ya que es la base por la que debe comenzar todo intento posible.

Los doctores Dorta Martínez y José Amado (2012) proponen una metodología basándose en los fundamentos didácticos abordados en el enfoque cognitivo, comunicativo y sociocultural para la enseñanza de la lengua materna y en particular para la enseñanza - aprendizaje de la construcción del texto escrito, por tanto considera las tres etapas de orientación, ejecución y control, teniendo en cuenta que tanto estas como los subprocesos de la producción textual, tienen carácter recursivos, es decir, cada uno de ellos puede tener momentos de realización y presencia, en otro.

Por tanto, la construcción del texto escrito tiene carácter procesal el cual se estructura, a su vez, en tres subprocesos (planeación, textualización y autorrevisión) coincidentes con las etapas didácticas para su enseñanza (orientación, ejecución y control). Los marcadores textuales y del discurso, tienen gran importancia para el logro de la pertinencia antes mencionada. Antonio Nogueira Messias da Silva en su artículo publicado bajo el título de:

Los marcadores del discurso y su introducción en los manuales de E/LE nos ofrece las razones por las cuales se deben estudiar estas partículas como él también las define en su material y plantea que:

- Aunque los marcadores discursivos sean «denotativamente» superfluos, es evidente e indudable que también cumplen diversas funciones discursivas que, como otras palabras de valor referencialmente reconocido, ayudan a construir el sentido del discurso (Martín Zorraquino, 2004, p. 56). 
- Y si desempeñan tales funciones, constituyen parte de la competencia comunicativa de los hablantes, la cual, actualmente en las clases de Lengua Extranjera, se intenta que los estudiantes adquieran para aprender bien la lengua meta (Martín Zorraquino, 2004, p. 56).

- Los aprendices de una lengua extranjera deben conocer y practicar, además de las reglas de la gramática de esa lengua extranjera, otras importantes reglas responsables de la construcción de diferentes géneros discursivos; y si, de hecho, quieren obtener una competencia comunicativa plena, también deberán dominar las reglas que regulan la interacción entre los interlocutores y el contexto, para todo lo cual el dominio del uso adecuado de los marcadores discursivos es imprescindible (Martín Zorraquino, 2004, p. 56).

- En vista de que los conectores discursivos pertenecen a un grupo de elementos que, con diversas acepciones o matices, son muy frecuentes en los distintos géneros discursivos, es necesario que el aprendiz conozca bien sus propiedades distribucionales, semánticas y pragmáticas para que pueda, con mayor facilidad y éxito, producir y entender los diferentes discursos.

- El conocer bien esas propiedades implicará, por consiguiente, el conocimiento de las características de un considerable conjunto de partículas necesarias para la construcción del discurso, así como la expresión de la subjetividad del hablante y, entre otras cosas, diversos y sutiles matices de expresividad de la lengua meta.

- El uso intencional de los conectores discursivos por el estudiante significa que desea incrementar el acceso al contexto y la relevancia de su discurso, así como que su oyente obtenga las inferencias pertinentes de los enunciados que profiere aquel.

- El dominio de los conectores discursivos favorece la articulación del discurso de los aprendientes, pues con estas piezas ellos dan a entender con mayor agilidad y elegancia sus ideas, pensamientos o representaciones del mundo. 
- Por último, ha de tenerse presente que el uso marginal de los conectores discursivos, tanto en los textos escritos como en los orales, es responsable de guiar erróneamente las interpretaciones de ciertos contenidos informativos y, por ende, de conducir al interlocutor (lector u oyente) inexperto a una comprensión equivocada (Nogueira, 2012, p.6).

Ileana Domínguez hace alusión a dos cualidades del párrafo como: la coherencia y el énfasis, se dice que: "Un texto es coherente si el proceso de comunicación las personas son capaces de encontrarle sentido y de asignarle una organización a sus partes, por ello la coherencia es, ante todo, un fenómeno mental” (Domínguez, 2012 p. 206).

Es decir, que la errónea estructuración de elementos gramaticales que constituyen conectores textuales, muchos de los cuales se emplean para establecer las relaciones entre una y otra expresión, son los causantes de la pérdida en el texto de la coherencia, la cohesión y el énfasis. Por tanto, se logrará un adecuado texto escrito siempre y cuando un grupo de oraciones tenga sentido global, o sea; que constituyan un texto bien formado, para ello, deben tener un tema central que una la secuencia de oraciones.

Por otro lado, la coherencia local que solemos encontrar en todo texto construido se da a través de las relaciones que se establecen entre una oración y otra propiciando relaciones causales y condicionales entre otras. Estas se enmarcan con elementos gramaticales que sirven de nexo o de conectores permitiendo la cohesión o coherencia formal. Estos elementos que expresan relaciones lógicas entre las oraciones de un texto son: las conjunciones, preposiciones, pronombres, adverbios y frases conjuntivas.

En el libro de "Español-Literatura para escuelas pedagógicas, tomo II", de los doctores Ana María Abello Cruz y Juan Ramón Montaño Calcines encontramos el siguiente criterio:

Hoy día consideramos como errores que atentan contra la coherencia el uso indebido de pronombres, preposiciones y conjunciones, es decir, la errónea estructuración de elementos gramaticales que constituyen conectores textuales, muchos de los cuales se 
emplean para establecer relaciones entre las expresiones que conforman el texto escrito. (Abello Cruz y Montaño Calcines, 2012, p.106)

En el libro Gramática Española Contemporánea de la Gramática de la Lengua a la Gramática del Discurso I, de la doctora Alicia Toledo Costa y otros autores hacen una caracterización pragmática de las preposiciones y las conjunciones reconociéndolos como conectores del discurso. (Toledo Costa y otros, 2014). Otros gramáticos como Daniel Hernández Ruiz y Tatiana Gunko plantean que los marcadores discursivos o conectores del discurso son: “elementos muy importantes para tener un buen nivel de español tanto al escribir como al hablar. Estas partículas sirven para ordenar y conectar las distintas partes del discurso facilitando la cohesión textual y la interpretación de los enunciados” (Hernández, Ruiz y Gunko, 2014).

Ellos proponen de manera online algunos ejercicios de práctica. La Dra. Mildrey Clavel Meralla (2015) en el libro de Orientaciones Metodológicas sobre el Programa Director de la Lengua Materna expone los objetivos generales del mismo, dentro de ellos y relacionado con el tema que nos ocupa son: "Expresarse de forma creadora mediante la construcción de textos de diferente tipología, desarrollar las habilidades comunicativas, mediante el empleo de estrategias de comprensión y construcción de textos coherentes en diferentes estilos" (Clavel Meraya, 2015, p. 6).

Según Beaugrande, las características de la textualidad son: cohesión, coherencia, intencionalidad, aceptabilidad, informatividad, situacionalidad, intertextualidad. ¿Y qué relación establece la gramática con estas características de la textualidad? ¿Qué importancia tiene que los estudios gramaticales tengan en cuenta estas características? A través de la gramática tradicional se le ha estado tratamiento al contenido mediante ejercicios de reconocimiento y clasificación de elementos que sirven para relacionar, con esta terminología aparecen en los temarios ejercicios vinculados solo a nivel de reconocimiento. 
Las obras de autores como: Teun A. Van Dijk, Rafael Núñez y Enrique del Teso, Marina Parra, Gisela Cárdenas Molina, Magdalena Viramonte de Avalos, Felipe Zayas, Manuel Casado Velarde y Angelina Roméu Escobar ofrecieron en sus obras aportes teóricos y didácticos con respecto a la construcción del discurso, ellas contienen definiciones de las dos categorías funcionales del texto: la coherencia y la cohesión; además de incluir el sistema de conocimientos de la lengua que conllevan al logro de las cualidades del discurso.

Desde la didáctica de la lengua, con un enfoque cognitivo, comunicativo y sociocultural, la construcción del texto escrito deviene en interés para los docentes de forma general, especialmente para los de Español-Literatura, debido a la importancia que en el proceso de enseñanza-aprendizaje tiene el lenguaje, para ello se tienen en cuenta de forma interrelacionada, los factores socioculturales, emotivos o afectivos, cognitivos, físicos, discursivos, semánticos, pragmáticos y verbales (Roméu, 2016).

Por otra parte, el M. Sc. José Alberto López Díaz en su libro “Compendio de gramática española y apuntes sobre redacción", nos dice que:

Todo texto busca ser pertinente y esto se logra de forma gradual al introducirse las ideas pues, una conduce a la otra y el emisor debe utilizar adecuadamente las llamadas pistas o marcas según su intención comunicativa en el momento de la redacción pues, si va a presentar la introducción, si va a pasar al desarrollo, si va elaborar una nueva idea, si va a sumar otro argumento a lo dicho, si va a cambiar de criterio, si desea hacer hincapié, detallar, resumir y concluir deberá hacer un adecuado uso de los conectores y marcadores textuales tales como: las conjunciones, adverbios, locuciones prepositivas o conjuntivas e incluso frases, pues estas servirán para darle estructura al texto estableciendo un orden y relaciones significativas entre sus partes así como para estructurar las ideas dentro del texto conectando las ideas entre sí y formando las oraciones que integrarán el párrafo. (López Díaz, 2017, pp.84-85) 
La gramática tradicional no se interesó por estas construcciones, pero una gramática del discurso no puede soslayarlas. Una de las características de la textualidad muy importante es la informatividad. Precisamente la función informativa es la que posibilita valorar si las estructuras gramaticales tales como el sujeto, el complemento directo, indirecto y las clases léxico-sintácticas de palabras aportan al discurso, o sea, determinan si la información es nueva o no, si hay alguna estructura gramatical que la ponga de relieve.

Es importante mencionar que el trabajo realizado por los doctores Miguel A. Rodríguez Aragón y Miriam Dorta Martínez (2019) en los que diseñaron una estrategia metodológica para contribuir a la solución de algunos problemas de la construcción de textos desde la concepción lingüística textual que sirvió de base para el proceso de enseñanza-aprendizaje de la construcción del discurso para elevar la calidad del proceso enseñanza-aprendizaje de la construcción del texto enfatizaron no solo en los textos literarios sino también en los no literarios, esto favorece las relaciones interdisciplinarias con otras materias lectivas, cierto es que; el docente en el aula le brinda mayor interés a la creación de textos literarios por las características que posee esta tipología textual, dada por la incorporación de lo ético, lo estético, así como el desarrollo de la competencia literaria crítica y creadora.

Al respecto, el Dr. Juan Ferrer Gardona en su tesis en opción al grado científico, hace un profundo análisis de esta problemática y plantea la necesidad de la enseñanza gramatical a la luz de los postulados de la lingüística textual, en que cada una de las estructuras sean analizadas desde el funcionamiento de sus usos comunicativos, de manera que se aprovechen sus potencialidades para la comprensión, el análisis y la construcción de textos escritos.

Cabe entonces preguntar: ¿Por qué se ha limitado el estudio de los conectores discursivos solo al plano gramatical? ¿Por qué se trabajan en la educación preuniversitaria desde el reconocimiento y clasificación según la función que ocupa en el texto? ¿Por qué no trabajar la construcción del texto escrito desde varios enfoques comunicativos enfatizando en el uso correcto de estos enlaces oracionales? 
Pues, teniendo en cuenta los criterios expresados por varios gramáticos podemos resumir que el estudio de los conectores o marcadores discursivos se ha convertido en una prioridad a partir del desarrollo de la lingüística del texto, de la gramática del discurso y de la pragmática, pues, no puede haber creación coherente en un texto, ya sea de forma oral o escrita sin su empleo; ya que ellos nos sirven de guía a la hora de establecer una adecuada comunicación y como se dijo anteriormente están constituidos por: conjunciones, preposiciones, adverbios, locuciones conjuntivas, sintagmas o expresiones lexicalizadas que actúan en el texto para relacionar todas las ideas ya sean oraciones o párrafos, mostrando las conexiones y partes del discurso, esto contribuye a que el texto obtenido tenga carácter coherente y unitario.

Por eso, la Educación Preuniversitaria debe transformar el proceso de enseñanza-aprendizaje de la construcción del texto escrito, manteniendo los tres momentos cruciales que todos conocemos: la orientación, la ejecución y el control, pero, haciendo especial énfasis en cómo saber emplear los conectores y marcadores del discurso según la forma elocutiva solicitada a los educandos.

\section{Propuesta del sistema de ejercicios para el empleo de los conectores discursivos en la construcción del texto escrito en la asignatura español-Literatura en el Preuniversitario.}

En cada uno de ellos hay ejercicios que van del primer nivel de asimilación del contenido hasta el segundo y tercer nivel que es el aplicativo y extrapolativo, en ellos se han insertado los ejercicios de reconocimiento, aplicación y extrapolación de los conectores discursivos.

1- Lee detenidamente el siguiente texto y realiza las actividades propuestas:

La enfermedad coronaria es más frecuente, más precoz y más severa en el diabético, la protección conferida por los estrógenos se pierde en la mujer menopáusica, igualándose el riesgo cardiovascular a la del hombre de la misma edad. El riesgo de enfermedad coronaria en el hombre es el doble y hasta cinco veces más alto en la mujer diabética cuando se compara con la no diabética. Algunos de los factores de riesgo de enfermedad cardiovascular como la 
obesidad, la hipertensión y la dislipidemia son más frecuentes y a un mismo grado de severidad predicen mayor riesgo de eventos cardiovasculares en la persona con diabetes.

a) Estamos en presencia de un texto: literario o no literario.

b) La intención comunicativa del autor es:

_ Informar al lector sobre la enfermedad coronaria.

_ _ Informar al lector sobre la enfermedad coronaria y las personas más afectadas. Informar al lector de las causas que agilizan la enfermedad coronaria.

c) Extraiga del texto dado cinco conectores o marcadores del discurso. Especifique su tipo.

2- Lee detenidamente el siguiente texto y luego subraya el conector adecuado según convenga, cuidado porque hay conectores que tienen homófonos:

(A-Al) llegar a la más occidental (de-en) nuestras provincias, conocerá una tradición (quéque) nace (dé-de) la escogida de la hoja de tabaco (hasta-asta) la final elaboración del habano. Una cultura, color y aroma (que-qué) recorre el mundo.

a) ¿Te gusta el café? Construye un texto que no se exceda de un párrafo y que tenga carácter expositivo, en el que expreses tu criterio al respecto. Ten en cuenta para su realización el empleo adecuado de los conectores o marcadores discursivos.

3- Lee atentamente el siguiente texto y luego realiza las actividades propuestas: Saber cómo se formó nuestro planeta y cómo apareció la vida en este, ha sido siempre una obsesión para los hombres. Los pueblos antiguos, desconocedores de la ciencia y de los grandes descubrimientos que esta haría siglos después, trataban de dilucidar lo que consideraban un misterio, mucho antes de que se aceptara por toda la teoría de la redondez de la Tierra. Los griegos, por su parte, concibieron la genealogía de sus dioses y con esta trataron de explicarse la gran incógnita.

a) La idea central del texto es:

El surgimiento de la Tierra.

La vida en el planeta Tierra.

El interés de los hombres hacia el conocimiento de la formación de la Tierra y sus habitantes. 
b) Extraiga del texto inicial cinco conectores o marcadores discursivos que difieran en clasificación. Especifique su tipo en cada caso.

4- Completa el siguiente texto con los conectores o marcadores discursivos que te damos a continuación: (además, si no, y por ello, de, y, que)

La educación física es parte integral _ la cultura del hombre , el concepto

persona culta debe comprender, del grado de instrucción educación, el nivel desarrollo físico. La carencia del ejercicio dosificado _ necesario es la causa de la casi totalidad _ las enfermedades vasculares. Por muy altas sean las cumbres del saber humano, de nada valen su cultura _ sus conocimientos posee lo principal: la salud.

5- Lee detenidamente el siguiente texto y relacionado con él realiza las actividades propuestas: En las postrimerías del siglo XIX e inicios del siglo XX, surgió la nueva trova cubana, de la cual Silvio Rodríguez y Pablo Milanés son los exponentes más conocidos. Se basó principalmente en la composición para guitarra y voz. Las letras por lo general mostraban un contenido social relacionado con la revolución socialista de 1959. En otros países latinoamericanos, la palabra "trova" se refiere a estilos de música que pueden no estar relacionados con la trova caribeña. Generalmente el término "trovador" se refiere al músico popular dentro de las canciones de protesta, incluso en género rock.

a) Marca con una (X) la respuesta correcta:

b) - La forma elocutiva empleada en el texto inicial es: narración exposición descripción

c)La intención comunicativa del texto es:

Dar una orden Ofrecer una información Sugerir varias ideas.

6- Complete los siguientes enunciados con sintagmas nominales a partir del conocimiento que le proporciona la lectura del texto dado:

La primera trova fue escuchada en___, específicamente en la región , cuyo tema principal fue la 
7- Completa el siguiente texto con los conectores discursivos que te damos a continuación y las tildes que faltan: (y, por lo general, entre, pero, con, como) sus temas predilectos figuran el amor, la caballería, la religión, la política, la guerra, los funerales _ la naturaleza. Sus formas de versificación eran la canción de amor), la tensión (diálogos _ debates), el serventesio (canción política o satírica), el planto (canto fúnebre o endecha), el alba (canción matinal) y la serena (canción nocturna). El acompañamiento musical se interpretaba generalmente instrumentos de cuerda el violín medieval o el laúd. La notación indicaba el tono, no el tempo o el ritmo.

8- De las canciones que has escuchado de la nueva trova y los conocimientos que obtuviste en el grado acerca de este género musical, piensa en la que más te haya gustado y en un texto que no exceda los dos párrafos expresa el porqué de tu elección. No olvides hacer un buen uso de los conectores o marcadores discursivos.

9- En el siguiente cuadro aparecen mezclados algunos conectores o marcadores del discurso, encuéntralos y extráelos:

\begin{tabular}{|l|l|l|l|l|l|l|l|l|l|l|}
\hline c & o & n & r & e & s & p & e & c & t & o \\
\hline o & c & o & a & n & i & e & s & a & y & i \\
\hline n & a & o & z & c & n & n & d & p & d & e \\
\hline t & s & b & o & u & o & s & e & a & i & n \\
\hline s & i & n & n & a & u & o & c & z & i & l \\
\hline s & o & t & a & n & t & o & i & s & l & a \\
\hline o & n & a & n & t & p & e & r & o & m & b \\
\hline b & e & n & a & o & a & n & p & s & i & r \\
\hline r & s & t & r & a & r & i & o & o & s & i \\
\hline e & z & e & e & n & a & u & r & s & u & l \\
\hline
\end{tabular}

10- Completa con los conectores discursivos que a continuación te ofrecemos el siguiente texto: (de, en, a, con, mientras que, y, que, para) 
La belleza desbordada _ las letras de Neruda, sentimientos encontrados _ _ nos dejan siempre que leemos su maravillosa obra, vasta experiencia _ la vida. No quedó un elemento de la naturaleza al __ él no le brindara homenaje, pienso que, cada cosa que nos rodea formó parte __ su pensamiento ___ luego crear, __ minuciosidad un espléndido poema.

11- Coloque los conectores discursivos que faltan y subraye el grafema correcto en el vocablo que lo indica: (entre, en, y, de, que)

$\mathrm{Su}$ sentimentalismo es grandilocuente _ desafiante, en lugar de ser recatado y retraído, colocándose __ las primeras de las devoluciones radicales. Lejos _ ser una aberración, Dion _ realidad es símbolo de una clase de pop sensible, en donde más grande es mejor, donde dema(s-c)iado nunca es suficiente, y en donde la más madura emoción es muy cierta. Su voz se desliza fácilmente desde profundos su(s-z)urros hasta las notas muertas más altas, una dulce sirena __ combina la fuerza con la gracia. El volumen, como tal, es delgado, pero gracias a su perfecto dominio del respaldo na(s-z)al, su voz adquiere un timbre inci(s-c)ivo y penetrante, con una gran rique(z-s)a armónica.

12- De todos los artistas que conoces de tu país o del resto del mundo, escoge el que prefieras y construye un texto que no exceda los dos párrafos refiriéndote a sus características, el género musical que cultiva, así como las temáticas que aborda en sus piezas.

\section{Conclusiones}

La aplicación del sistema de ejercicios propició elevar la calidad del proceso de enseñanzaaprendizaje de la asignatura español-Literatura en cuanto al uso de los conectores discursivos en la construcción de textos escritos, así como el nivel cultural de los educandos.

\section{Referencias}

Abello Cruz, A. M. y Montaño Calcines, J. R. (2012): Español-Literatura para escuelas pedagógicas. La Habana, Cuba: Ed. Pueblo y Educación, p.106 
Clavel Meralla, M. (2015). Orientaciones Metodológicas. Programa Director de Lengua Materna. Educación Preuniversitaria. La Habana, Cuba: Ed. Pueblo y Educación, p.6

Domínguez García, I. (2010). Comunicación y texto. La Habana, Cuba: Ed. Pueblo y Educación.

Domínguez García, I. y coautores (2012). Lenguaje y comunicación. La Habana, Cuba: Ed. Pueblo y Educación, p.206

Ferrer Gardona, J. (1999). Modelo de Rediseño de los Contenidos Gramaticales en Función de la Competencia Comunicativa. La Habana, Cuba: Ed. Pueblo y Educación. pp. 41-42.

Hernández Ruiz, D. y Gunko, T. (2014). Marcadores discursivos o conectores del discurso. Recuperado de ProfeDeELE.es. Descargado por Google.

López Díaz, J. A. (2018). Compendio de gramática española y apuntes sobre redacción. La Habana, Cuba: Ed. Pueblo y Educación. pp. 84-85

Mañalich, R., Cancio, C., Echemendía, D. M. y Basso, Z. (2004). Taller de la Palabra (3ra reimpresión). Habilidades de expresión escrita: enfoque comunicativo-motivacional. Ciudad de La Habana, Cuba: Ed. Pueblo y Educación. pp. 206-220.

Mañalich, R., García, C. y Cuéllar, M. A. (2004). Taller de la Palabra (3ra reimpresión).El desarrollo de habilidades en la construcción de párrafos. Ciudad de La Habana, Cuba: Ed. Pueblo y Educación. pp. 193-205.

Mañalich, R. y Hernández, J. E. (2004). Taller de la Palabra (3ra reimpresión). Las transformaciones y condiciones de la coherencia semántica y la redacción de textos. Ciudad de La Habana, Cuba: Ed. Pueblo y Educación. pp. 177-184. 
Martín Zorraquino, M. a Antonia. (1991). "Elementos de cohesión en el habla de Zaragoza", en José M. ${ }^{a}$ Enguita Utrilla (ed.), I Curso de Geografía Lingüística de Aragón, Zaragoza, Institución Fernando el Católico: 253-286.

(1992): «Partículas y modalidad», en Günter Holtus et al. (eds.), Lexikon der romanistisches Linguistik, Tubinga, Max Niemeyer Verlag, VI (1): 110-124.

(1994): “Gramática del discurso. Los llamados marcadores del discurso”, en Actas del Congreso de la Lengua Española, Sevilla, 7 al 10 de octubre de 1992, Madrid, Instituto Cervantes: 709-720.

(1994): "Bueno" como operador pragmático en español actual", en Alegría Alonso et al. (eds.), II Encuentro de Lingüistas y Filólogos de España y México, Salamanca, Junta de Castilla y León/Universidad de Salamanca: 403-412.

(1994): “Sintaxis, semántica y pragmática de algunos adverbios oracionales asertivos en español actual”, en Violeta Demonte (ed.), Gramática del español, México, El Colegio de México: 557-590.

Nogueira Da Silva, A. M. (3 de agosto de 2012): Los marcadores del discurso y su introducción en los manuales de E/LE. Universidade Federal do Pará (Brasil). Recuperado de Google. p.6

Roméu Escobar, A. y coautores (2016). Didáctica de la lengua española y la literatura. Tomo II. (pp.1, 20, 66,100) La Habana, Cuba: Ed. Pueblo y Educación.

Toledo Costa, A. y otros (2014): Gramática española contemporánea. De la gramática de la lengua a la gramática del discurso. La Habana, Cuba: Ed. Pueblo y Educación. 
REVISTA INTERNACIONAL DE PEDAGOGÍA E INNOVACIÓN EDUCATIVA |

Volumen 1. Número 1. Enero - Junio 2021

ISSN: 2745-0341 (En línea) 